\title{
Relational structures for measures of ignorance
}

\author{
Camilo Franco, Javier Montero, J. Tinguaro Rodríguez
}

Faculty of Mathematics, Complutense University, Madrid

\begin{abstract}
Different types of inexactness can be represented by fuzzy sets: vagueness, where there are no precise boundaries, ambiguity, when more than one distinguishable concept is described, generality, such that a word applies to a variety of situations, and ambivalence, where conflicting valuations can coexist. Different measures of ignorance can be used in order to grade the distinct attributes of inexact information. Here we explore some of these measures from a relational point of view.
\end{abstract}

Keywords: Ambiguity, Ambivalence, Vagueness, Fuzziness, Ignorance, Relational structures

\section{Introduction}

The examination of ignorance as an epistemic state [3],[9] often considers ignorance in its most general form, as the absence of knowledge. But the concept of ignorance has many faces. The complexity of understanding ignorance involves on one hand, its representation, and on the other, its different manifestations.

In its most general form, ignorance as absence of knowledge can be understood as a primary type of uncertainty [13], while other manifestations like e.g., false belief, vagueness, inexactness, ambiguity or ambivalence, seem of a more specialized type, more likely belonging to a more elaborated state of mind. Basically, ignorance, as primary uncertainty, implies all other manifestations, but all other manifestations do not imply lack of knowledge. This was an underlying argument in [9], where ignorance was associated to a first stage of knowledge, previous to any kind of measurement.

The intuition here is that the simplest epistemic attribution for ignorance refers to the absence of relevant knowledge, such that the word ignorance refers to a situation where there is no precise knowledge about a set of objects of interest [15]. Under this view, ignorance can be classified into two epistemic categories, one concerned with the validity of information, i.e., uncertainty, and the other concerned with the exact meaning of information, i.e., ambiguity.

In this paper we explore distinct notions of ignorance, such as ambiguity, ambivalence, vagueness and generality, examining their differences and similarities, and showing that a number of measures used to study ignorance satisfy the conditions that characterize ambiguity relational structures. In order to do so, we now introduce relational structures as it has been done in [15]. For some universe $X$, where $P(X)$ is the set of all subsets of $X$, i.e., the power set of $X$, and $\neg A$ stands for the complement of $A \in P(X)$, let $\succ$ be a relation on
$P(X)$ and $f$ a numerical measure of ignorance, i.e., a function

$$
f: P(X) \rightarrow[0,1]
$$

The quantitative measure $f$ fully agrees with $\succ$ if, for all $A, B \in P(X)$,

$$
A \succ B \Leftrightarrow f(A)>f(B) .
$$

In this sense, $(P(X), \succ)_{f}$ is a relational structure defined by $f$, if this measure fully agrees with $\succ$. Notice if $\neg(A \succ B)$ and $\neg(B \succ A)$, then $A \sim B$, and if $A \sim B$ or $A \succ B$ then $A \succeq B$.

\section{Ambiguity}

A characterization for ambiguity functions has been given in [4], following some original insights from [7],[8]. Under this particular interpretation, an ambiguity function

$$
\alpha: P(X) \rightarrow[0,1]
$$

is a mapping satisfying the following axioms for all $A, B \in P(X)[4]:$

A1. $\alpha(\varnothing)=0, \alpha(A) \geq 0$

A2. $\alpha(A)=\alpha(\neg A)$,

A3. $\alpha(A \cap B)+\alpha(A \cup B) \leq \alpha(A)+\alpha(B)$.

Ambiguity is presented here as a comparative relation between sets or events $A, B$, such that $A \succeq B$ if and only if $A$ is at least as ambiguous as $B$. Notice that $A 1$ and $A 2$ imply that $\alpha(X)=0$. Condition $A 2$ states that ambiguity is the same for any set and for its complement, while condition $A 3$, expresses that the union of two sets $A, B$ may reduce or cancel ambiguities associated to each one (considered separately), but never increase them.

Ignorance due to ambiguity has been examined as an epistemic state that affects uncertain beliefs based on world knowledge (see e.g., [5]), in such a way that it stands out when subjects evaluate crisp-clear and vague prospects jointly. Consequently, ambiguity of the vague prospect greatly diminishes when it is evaluated in isolation. Under this perspective (referred to as the comparative ignorance hypothesis [5]), ambiguity is an inherently comparative effect representing the reluctance 
to act on inferior knowledge, a situation that becomes evident when comparing with superior knowledge. Thus, if it is possible to compare a vague event $A$ with a certain event $B$, the original intuition in $A 3$ [4] should be replaced by:

$$
\text { AC3. } \alpha(A \cap B)+\alpha(A \cup B) \geq \alpha(A)+\alpha(B) \text {. }
$$

But an absolutely certain event is not always available. Moreover, from a subjective viewpoint, the $a$ priori specification of an individual's state of knowledge is not always possible. Therefore, in case the state of knowledge regarding the events in question can be specified and a certain event is available, AC3 seems to be a good alternative to be taken into account when studying ambiguity in decision making contexts. Elsewhere, under more general and vague situations, the characterization of ambiguity as given by $A 1-A 3$ seems most appropriate.

Such characterization $A 1-A 3$ of ambiguity has been proved useful with respect to some concepts of Dempster-Shafer's theory of evidence [12],[15], as in the following example. A belief function is a mapping

$$
\mathrm{Bel}: P(X) \rightarrow[0,1]
$$

satisfying the following axioms, for every positive integer $n$ and every collection $A_{1}, A_{2}, \ldots, A_{n} \in X$ :

$\operatorname{Bel1} \operatorname{Bel}(\varnothing)=0$,

$\operatorname{Bel2} \operatorname{Bel}(X)=1$,

$\operatorname{Bel3.} \operatorname{Bel}\left(A_{1} \cup A_{2} \ldots \cup A_{n}\right) \geq \sum_{i} \operatorname{Bel}\left(A_{i}\right)-$

$$
\sum_{i<j} \operatorname{Bel}\left(A_{i} \cap A_{j}\right)+\ldots+(-1)^{n+1} \operatorname{Bel}\left(A_{1} \cap A_{2} \ldots \cap A_{n}\right)
$$

Given a belief function $\mathrm{Bel}$, its corresponding plausibility function, $P l$, is defined for all $A \in P(X)$, as

$$
\operatorname{Pl}(A)=1-\operatorname{Bel}(\neg A)
$$

Following [15], there exists a belief function satisfying the condition

$$
A \succ B \Leftrightarrow \operatorname{Bel}(A)>\operatorname{Bel}(B),
$$

i.e., Bel fully agrees with $\succ$, if and only if the relation $\succ$ satisfies the following axioms:

B1. $X \succ \varnothing$,

B2. $A \succ B \Rightarrow \neg(B \succ A)$,

B3. $\neg(A \succ B), \neg(B \succ C) \Rightarrow \neg(A \succ C)$,

B4. $A \supseteq B \Rightarrow \neg(B \succ A)$,

B5. $(A \supset B, A \cap C=\varnothing) \Rightarrow(A \succ B \Rightarrow A \cup C \succ B \cup C)$.
In the same way [15], there exists a plausibility function

$$
P l: P(X) \rightarrow[0,1]
$$

satisfying the condition

$$
A \succ B \Leftrightarrow P l(A)>P l(B),
$$

i.e., $P l$ fully agrees with $\succ$, if and only if the relation $\succ$ satisfies the following axioms:

P1. $X \succ \varnothing$,

P2. $A \succ B \Rightarrow \neg(B \succ A)$,

P3. $\neg(A \succ B), \neg(B \succ C) \Rightarrow \neg(A \succ C)$,

P4. $A \supseteq B \Rightarrow \neg(B \succ A)$,

P5. $(A \supset B, A \cup C=\varnothing) \Rightarrow(A \succ B \Rightarrow A \cap C \succ B \cap C)$.

Then, the function defined by

$$
\alpha(A)=P l(A)-\operatorname{Bel}(A)
$$

is an ambiguity function [15]. Hence, the greater the interval between plausibility (the amount of information that potentially supports $A$ ) and belief (the amount of information that surely supports $A$ ), the greater the ignorance due to ambiguity on $A$ there will be.

Notice that (1) can be rewritten as

$$
\alpha(A)=1-(\operatorname{Bel}(\neg A)+\operatorname{Bel}(A)),
$$

where the nature of ambiguity can be directly grasped, as everything that is not believed about $A$ or its complement $\neg A$. In other words, if there is ignorance on the boundaries of $A$, i.e., on the precise meaning of the concept represented by the set $A$, then there is presence of ambiguity. As a result, ambiguity easily resembles with the basic idea underlying fuzziness, as it focuses on the lack of distinction between a set $A$ and its complement or strong negation $\neg A[16]$.

The extension of ambiguity measures [4] to fuzzy set theory [17] is straightforward using traditional definitions for the complement of a set and intersection and union between sets [16]. For any fuzzy set $A$ defined on a set $X$, where the degree of membership of any $x \in X$ in $A$ is denoted by $A(x)$, taking its values from any partially ordered structure $L$ (see [6]), as e.g., $L=[0,1]$, ambiguity measures can be defined for any $A, B \in P^{f}(X)$, where $P^{f}(X)$ is the set of all fuzzy sets of $X$, as a function

$$
\alpha: P^{f}(X) \rightarrow[0,1]
$$

satisfying $A 1-A 3$, such that $A \cup B=A \vee B$, $A \cap B=A \wedge B$ and $\neg A=1-A$, where $\vee$ represents some continuous triangular conorm ( $t$-conorm) $S$, and $\wedge$ represents some continuous triangular norm ( $t$-norm) 
$T$ [11]. Some examples for $T$ and $S$ are, for any $x, y \in[0,1]$, the minimum and maximum,

$$
\begin{aligned}
& T(x, y)=\min (x, y), \\
& S(x, y)=\max (x, y),
\end{aligned}
$$

the product,

$$
\begin{gathered}
T^{\prime}(x, y)=x \cdot y, \\
S^{\prime}(x, y)=x+y-x \cdot y,
\end{gathered}
$$

or Lukasiewicz's,

$$
\begin{gathered}
T^{L}(x, y)=\max (x+y-1,0), \\
S^{L}(x, y)=\min (x+y, 1) .
\end{gathered}
$$

Following [4],[15], where crisp ambiguity relations have been defined, an ambiguity relation can be extended to a fuzzy environment as follows. Let $\succ$ be a relation on $P^{f}(X)$. There exists an ambiguity function

$$
\alpha: P^{f}(X) \rightarrow[0,1]
$$

for all $A, B \in P^{f}(X)$ satisfying the condition

$$
A \succ B \Leftrightarrow \alpha(A)>\alpha(B),
$$

i.e., $\alpha$ fully agrees with $\succ$, if and only if the relation $\succ$ satisfies the following axioms:

$A R 1 . \succ$ is a weak order (complete and transitive),

$A R 2 . A \succeq \varnothing$,

AR3. $A \sim \neg A$,

$A R 4$. If $\left(B_{1}, B_{2}, \ldots, B_{m}\right)$ is a rearrangement of

$$
\begin{aligned}
\left(A_{1} \wedge A_{2}, A_{1} \vee A_{2}, A_{3} \wedge A_{4}, A_{3} \vee A_{4}, \ldots\right. \\
\left.\ldots, A_{m-1} \wedge A_{m}, A_{m-1} \vee A_{m}\right)
\end{aligned}
$$

where $m$ is a positive integer and $A_{i} \in P^{f}(X)$, $i=1,2, \ldots, m$, then it is false that $B_{j} \succeq A_{j}$ for $j=1, \ldots, m$ and $B_{j} \succ A_{j}$ for at least one $j \in 1, \ldots, m$.

If $\succ$ satisfies $A R 1-A R 4$, then it is called an ambiguity relation and $\left(P^{f}(X), \succ\right)_{\alpha}$ is called an ambiguity structure.

\section{Remarks}

1. If we take into consideration the comparative ignorance hypothesis [5] expressed by $A C 3$, i.e., in case the state of knowledge regarding the events in question can be directly specified, condition $A R 4$ can be reformulated as:

$A R 4^{*}$. If $\left(B_{1}, B_{2}, \ldots, B_{m}\right)$ is a rearrangement of

$$
\left(A_{1} \wedge A_{2}, A_{1} \vee A_{2}, A_{3} \wedge A_{4}, A_{3} \vee A_{4}, \ldots\right.
$$

$$
\left.\ldots, A_{m-1} \wedge A_{m}, A_{m-1} \vee A_{m}\right),
$$

then it is false that $A_{j} \succeq B_{j}$ for $j=1, \ldots, m$ and $A_{j} \succ B_{j}$ for at least one $j \in 1, \ldots, m$.

2. Given the ambiguity structure $\left(P^{f}(X), \succ\right)_{\alpha}$, where fuzzy sets may describe more than one distinguishable concept, ambivalence can be examined where conflicting meanings or opposite interpretations coexist. Have in mind that ambiguity relations measure the extent in which an element $x$ can be both member of a set $A$ and its complement, being defined as a property for the pairs $\{A, \neg A\}$ of complementary sets on $P^{f}(X)$.

On the other hand, ambivalence refers to which extent an element $x$ can be both member of a set $A$ and its opposite. This is why ambivalence is a property defined for the pairs $\left\{A, A^{\mathrm{a}}\right\}$ of antagonistic sets on $P^{f}(X)$, where $A^{\mathrm{a}}$ is the antonym or opposite of the underlying concept represented by $A$ [9],[10],[14]. In this way, ambivalence can be examined by some measure defined for an antagonist type of negation, instead of a complemented or classical strong negation (i.e., a strictly decreasing and involutive function).

\section{Ambiguity, ambivalence and measures of fuzzi- ness}

Measures of fuzziness have been studied since [2], where a first characterization for measures of fuzziness, $\phi(A)$, is given for any $A, B \in P^{f}(X)$, such that $\phi(A)$ satisfies the following properties:

F1. $\phi(A)=0$ if and only if $A$ is a crisp set, i.e., $A(x)=0$ or $A(x)=1$.

F2. $\phi(A)$ has one maximal value for the fuzzy set $A$ such that $A(x)=0.5$ for all $x \in X$.

F3. If $A(x) \geq B(x) \geq 0.5$ or $0.5 \geq B(x) \geq A(x)$ then $\phi(A) \leq \phi(B)$.

Another suggestion for examining fuzziness focuses on the lack of distinction between a set $A$ and its complement or strong negation $\neg A$ [16]. If the intersection set $I$ is defined as

$$
I(x)=A(x) \wedge \neg A(x)
$$

then a measure of fuzziness, that also satisfies $F 1-F 3$, can be defined as [16],

$$
\phi^{Y}(A)=\frac{2}{n} \sum_{i=1}^{n} I\left(x_{i}\right)
$$


These two different approximations to fuzziness can be also studied through ambiguity measures (as it is shown in [16]).

Now, recalling our remark on ambivalence, a different kind of fuzziness can be examined if, instead of focusing on the lack of distinction between $A$ and its complement, we focus on the lack of distinction between $A$ and its opposite or antagonistic counterpart $A^{a}$ (for a detailed discussion on the semantics defined by opposite or polar terms the reader is referred to [10]). Therefore, if we define the intersection set as

$$
K(x)=A(x) \wedge A^{a}(x),
$$

a measure of polar-fuzziness can then be defined as,

$$
\psi^{a}(A)=\frac{2}{n} \sum_{i=1}^{n} K\left(x_{i}\right) .
$$

The extension from one kind of measure to the other is direct just by taking the set $A^{a}$ instead of $\neg A$, but the axioms characterizing the new measure have to be reconsidered.

The intuition behind this approach can be summarized by the following properties,

V1. $\psi(A)=0$ if and only if $A(x)=0$ or $A^{a}(x)=0$ for all $x \in X$.

$V 2$. $\psi(A)$ has one maximal value for the fuzzy set $A$ such that $A(x)=A^{a}(x)=1$ for all $x \in X$.

This type of measure can then be studied as an ambivalence measure.

Definition 1: An ambivalence measure is characterized as a function

$$
\psi: P^{f}(X) \times P^{f^{a}}(X) \rightarrow[0,1]
$$

where $P^{f^{a}}(X)$ is the set of all antagonistic fuzzy sets for $P^{f}(X)$, satisfying the following axioms for all $A, B \in P^{f}(X), A^{a} \in P^{f^{a}}(X):$

$A V 1 . \psi(\varnothing)=0$,

AV2. $\psi(A)=\psi\left(A^{a}\right)$,

AV3. $\psi(A \wedge B)+\psi(A \vee B) \geq \psi(A)+\psi(B)$.

Notice that the definition of ambivalence includes the comparative ignorance hypothesis, $A V 3$, in such a way that when two fuzzy sets representing different states of nature, i.e., fuzzy events, are compared, ambivalence tends to increase. Besides, if $\psi(A)=\psi(B)$, then $B=A^{a}$ and the inequality in $A V 3$ can be replaced by equality, such that

$$
\psi\left(A \wedge A^{a}\right)+\psi\left(A \vee A^{a}\right)=\psi(A)+\psi\left(A^{a}\right) .
$$

An ambivalence relation is characterized as follows.

Definition 2: Let $\succ$ be a relation on $P^{f}(X)$. There exists an ambivalence function

$$
\psi: P^{f}(X) \times P^{f^{a}}(X) \rightarrow[0,1]
$$

for all $A, B \in P^{f}(X), \quad A^{a} \in P^{f^{a}}(X)$ satisfying the condition

$$
A \succ B \Leftrightarrow \psi(A)>\psi(B)
$$

i.e., $\psi$ fully agrees with $\succ$, if and only if the relation $\succ$ satisfies the following axioms:

$A V R 1 . \succ$ is a weak order (complete and transitive), $A V R 2 . A \succeq \varnothing$,

AVR3. $A \sim A^{a}$,

$A V R 4$. If $\left(B_{1}, B_{2}, \ldots, B_{m}\right)$ is a rearrangement of

$$
\begin{aligned}
\left(A_{1} \wedge A_{2}, A_{1} \vee A_{2}, A_{3} \wedge A_{4}, A_{3} \vee A_{4}, \ldots\right. \\
\left.\ldots, A_{m-1} \wedge A_{m}, A_{m-1} \vee A_{m}\right),
\end{aligned}
$$

where $m$ is a positive integer and for $A_{i} \in P^{f}(X)$, $i=1,2, \ldots, m$, then it is false that $A_{j} \succeq B_{j}$ for $j=1, \ldots, m$ and $A_{j} \succ B_{j}$ for at least one $j \in 1, \ldots, m$.

If $\succ$ satisfies $A V R 1-A V R 4$, then it is called an ambivalence relation and $\left(P^{f}(X), P^{f^{a}}(X), \succ\right)_{\psi}$ is called an ambivalence structure.

\section{Ambiguity and interval structures}

An interval fuzzy structure $\left(F^{*}, F_{*}\right)$ is defined by a pair of lower and upper mappings representing the membership of the elements of a universe to a fuzzy set. In this sense, membership is characterized by an intervalvalued function and such characterization models an epistemic state commonly referred to as vagueness [3]. Following [15], where qualitative interval structures are defined, a lower mapping $F_{*}: P^{f}(X) \rightarrow[0,1]$ satisfies the following axioms for all $A, B \in P^{f}(X)$ :

$F_{*} 1 . F_{*}(\varnothing)=\varnothing$,

F*2. $F_{*}(X)=0$,

F 3. $F_{*}(A \wedge B)=F_{*}(A) \wedge F_{*}(B)$.

The corresponding upper mapping $F^{*}$ is defined by $F^{*}(A)=\neg F_{*}(\neg A)$, such that $F_{*}(A) \subseteq F^{*}(A)$ for all 
$A \in P^{f}(X)$. The upper mapping $F^{*}: P^{f}(X) \rightarrow[0,1]$ satisfies the following axioms for all $A, B \in P^{f}(X)$ :

$F^{*} 1 . F^{*}(\varnothing)=\varnothing$,

$F^{*}$ 2. $F^{*}(X)=1$,

$F^{*}$ 3. $F^{*}(A \vee B)=F^{*}(A) \vee F^{*}(B)$.

Theorem 1: Given the interval fuzzy structure $\left(F^{*}, F_{*}\right)$, the mapping $\alpha: P^{f}(X) \rightarrow[0,1]$ defined for all $A \in P^{f}(X)$ by,

$$
\alpha(A)=F^{*}(A) \wedge \neg F_{*}(A)
$$

is an ambiguity measure.

Proof: Axiom A1 follows directly from $F_{*} 1$ and $F^{*} 1$ :

$$
\alpha(\varnothing)=F^{*}(\varnothing) \wedge \neg F_{*}(\varnothing)=\varnothing
$$

Axiom A2 holds, by the definition of the upper mapping:

$$
\begin{aligned}
\alpha(\neg A) & =F^{*}(\neg A) \wedge \neg F_{*}(\neg A) \\
& =F^{*}(\neg A) \wedge F^{*}(A)=\neg F_{*}(A) \wedge F^{*}(A)=\alpha(A)
\end{aligned}
$$

Without any loss of generality, assume $A<B$, then, for $\wedge=\min$ and $\vee=\max$,

$$
\begin{aligned}
\alpha(A \wedge B) & =F^{*}(A \wedge B) \wedge \neg F_{*}(A \wedge B) \\
& =F^{*}(A) \wedge \neg F_{*}(A)=\alpha(A)
\end{aligned}
$$

and

$$
\begin{aligned}
\alpha(A \vee B) & =F^{*}(A \vee B) \wedge \neg F_{*}(A \vee B) \\
& =F^{*}(B) \wedge \neg F_{*}(B)=\alpha(B)
\end{aligned}
$$

hence axiom A3 holds,

$$
\alpha(A \wedge B)+\alpha(A \vee B)=\alpha(A)+\alpha(B)
$$

As a result, vagueness or imprecision, expressed by interval fuzzy structures, can also be examined through ambiguity measures.

Theorem 2: The mapping $\alpha: P^{f}(X) \rightarrow[0,1]$ defined for all $A \in P^{f}(X)$ by,

$$
\alpha(A)=\frac{1}{n} \sum_{i=1}^{n} F^{*}\left(A\left(x_{i}\right)\right)-F_{*}\left(A\left(x_{i}\right)\right)
$$

where $n$ is the cardinality of $X$, is an ambiguity measure (this expression is the indetermination index used for measuring entropy for interval valued fuzzy sets [1]).

Proof: Axiom A1 follows directly from $F_{*} 1$ and $F^{*} 1$ :

$$
\alpha(\varnothing)=\frac{1}{n} \sum_{i=1}^{n} F^{*}\left(\varnothing\left(x_{i}\right)\right)-F_{*}\left(\varnothing\left(x_{i}\right)\right)=\varnothing .
$$

Axiom A2 holds, by the definition of the upper mapping:

$$
\begin{aligned}
\alpha(\neg A) & =\frac{1}{n} \sum_{i=1}^{n} F^{*}\left(\neg A\left(x_{i}\right)\right)-F_{*}\left(\neg A\left(x_{i}\right)\right) \\
& =\frac{1}{n} \sum_{i=1}^{n} \neg F_{*}\left(\neg \neg A\left(x_{i}\right)\right)-F_{*}\left(\neg A\left(x_{i}\right)\right) \\
& =\frac{1}{n} \sum_{i=1}^{n} 1-F_{*}\left(A\left(x_{i}\right)\right)-F_{*}\left(\neg A\left(x_{i}\right)\right) \\
& =\frac{1}{n} \sum_{i=1}^{n} 1-F_{*}\left(\neg A\left(x_{i}\right)\right)-F_{*}\left(A\left(x_{i}\right)\right) \\
& =\frac{1}{n} \sum_{i=1}^{n} \neg F_{*}\left(\neg A\left(x_{i}\right)\right)-F_{*}\left(A\left(x_{i}\right)\right) \\
& =\frac{1}{n} \sum_{i=1}^{n} F^{*}\left(A\left(x_{i}\right)\right)-F_{*}\left(A\left(x_{i}\right)\right)=\alpha(A) .
\end{aligned}
$$

Without any loss of generality, assume $A\left(x_{i}\right)<B\left(x_{i}\right)$, then, for $\wedge=\min$ and $\vee=\max$,

$$
\begin{aligned}
& \alpha\left(A\left(x_{i}\right) \wedge B\left(x_{i}\right)\right)= \\
& =\frac{1}{n} \sum_{i=1}^{n} F^{*}\left(A\left(x_{i}\right) \wedge B\left(x_{i}\right)\right)-F_{*}\left(A\left(x_{i}\right) \wedge B\left(x_{i}\right)\right) \\
& =\frac{1}{n} \sum_{i=1}^{n} F^{*}\left(A\left(x_{i}\right)\right)-F_{*}\left(A\left(x_{i}\right)\right)=\alpha\left(A\left(x_{i}\right)\right)
\end{aligned}
$$

and

$$
\begin{aligned}
\alpha(A & \left.\left(x_{i}\right) \vee B\left(x_{i}\right)\right)= \\
& =\frac{1}{n} \sum_{i=1}^{n} F^{*}\left(A\left(x_{i}\right) \vee B\left(x_{i}\right)\right)-F_{*}\left(A\left(x_{i}\right) \vee B\left(x_{i}\right)\right) \\
& =\frac{1}{n} \sum_{i=1}^{n} F^{*}\left(B\left(x_{i}\right)\right)-F_{*}\left(B\left(x_{i}\right)\right)=\alpha\left(B\left(x_{i}\right)\right)
\end{aligned}
$$

hence axiom A3 holds,

$$
\begin{aligned}
& \alpha\left(A\left(x_{i}\right) \wedge B\left(x_{i}\right)\right)+\alpha\left(A\left(x_{i}\right) \vee B\left(x_{i}\right)\right)= \\
& \alpha\left(A\left(x_{i}\right)\right)+\alpha\left(B\left(x_{i}\right)\right)
\end{aligned}
$$

As it has been seen, ambiguity measures are valid for measuring entropy (theorem 2), as they are also valid for measuring the type of generality and vagueness characteristic of interval structures [15] and interval fuzzy sets (theorem 1). 


\section{Ignorance and ambiguity}

Recently (see [1]), a characterization for ignorance has been given for any $x, y \in[0,1]$ by a function

$$
G:[0,1]^{2} \rightarrow[0,1]
$$

that satisfies the following conditions:

$$
\begin{aligned}
& \text { G1. } G(x, y)=G(y, x) \text { for all } x, y \in[0,1], \\
& \text { G2. } G(x, y)=0 \text { if and only if } x=1 \text { or } y=1, \\
& \text { G3. If } x=0.5 \text { and } y=0.5 \text {, then } G(x, y)=1, \\
& \text { G4. } G \text { is decreasing in }[0.5,1]^{2}, \\
& \text { G5. } G \text { is increasing in }[0,0.5]^{2} .
\end{aligned}
$$

This function has the purpose of modeling the lack of knowledge when determining the membership degrees of some pixels of an image $Q$ to the fuzzy set representing the background of the image, $Q_{B}$, and to the fuzzy set representing the object in the image $Q_{O}$. Although this approach [1] was originally designed for recognizing pixels in an image, where the pixel either belongs to the background or to the object, the ignorance measure $G$ can be examined as a general ignorance function for any pair of fuzzy sets.

Axiom $G 1$ characterizes $G$ as a symmetric function, where ignorance does not depend on which membership, to the background $\left(Q_{B}\right)$ or to the object $\left(Q_{O}\right)$, is considered first. The second axiom, $G 2$, refers to the representation of complete knowledge as the situation where at least one of the membership intensities is 1 . The third axiom, G3, states that if the quantification of the expert knowledge that the pixel with intensity $q$ belongs to the background is $\mu_{Q_{B}}(q)=0.5$ and to the object is $\mu_{Q_{o}}(q)=0.5$, then total or maximum ignorance exists.

The two axioms $G 2$ and $G 3$ characterize $G$ as a function that examines the exact meaning of the information, where complete knowledge and complete ignorance are defined, and G3-G5 assure that $G$ has a maximum in 0.5. Therefore, if $x$ and $y$ are $1(x=y=1)$, then $G$ is 0 , but if $x$ and $y$ are $0(x=y=0)$ then $G$ is any value less than 1 . Notice that if such value is 0 , then $G$ holds a direct relation with measures of fuzziness $(F 1-F 3)$, in the sense that if $x=y=0$ then $\phi(x)=\phi(y)=0$.

But the model for which $G$ is originally thought for (its field of application and purpose is to binarize an image) considers the situation of perfect or total knowledge as the case where membership of a pixel (e.g., to $Q_{B}$ ) is null if and only if the other membership (e.g., to $Q_{O}$ ) is maximum. In such case, $G$ should return 0 only in the case where just one of the membership values is 0 (justification behind the formal definition of $G$ [1]). This condition, different from $G 2$, implies that if one of them is 0 , then the other has to be 1 , or in other case there is presence of ignorance, up to a certain degree.
Now, notice that an ambiguity measure (in the same way as a measure of fuzziness) returns 0 when, for any element $x \in X, A(x)=0$ or $A(x)=1$. In this sense, the binary restriction commented above can be added to the characterization of the ignorance function, $G$, using ambiguity measures, for any pair of fuzzy sets $A, B$, such that $G(A(x), B(x))=0$ if and only if $\alpha(A(x))=0, \alpha(B(x))=0$ and $A(x)=1-B(x)$.

Following the classification of ignorance into uncertainty and ambiguity [15], the ignorance function $G$ seems to fall directly under the second category, as it measures the exact meaning of the available information. But apart from the cases considered by such ignorance function, there exist other cases where the inexactness of information expresses distinct states of knowledge. Such cases represent different expressions of ignorance, as e.g., when there is no available information [9] (case where $A(x)=B(x)=0$ ) or when there are conflicting pieces of information (ambivalent case where $A(x)=B(x)=1)$.

\section{Final remarks}

As an expression of inexactness, ambiguity is related with vagueness, generality and fuzziness, among others. Under this approach, it represents a certain kind of ignorance over the exact meaning of information, different from the other kind of ignorance over the validity of the information, referred to as uncertainty. This classification is useful for distinguishing different attributes of ignorance, examining their similarities and also their differences. For any model it is of interest to deal with one or the other type of ignorance, but notice that ignorance is a complex epistemic state that has many faces, and as such, can be classified in different ways. This paper has shown that ambiguity relations are useful to properly identify some of ignorance's faces.

\section{References}

[1] H. Bustince, M. Pagola, E. Barrenechea, J. Fernandez, P. Melo-Pinto, P. Couto, H. Tizhoosh, J. Montero. Ignorance functions. An application to the calculation of the threshhold in prostate ultrasound images. Fuzzy Sets and Systems 161, 20-36, 2010.

[2] A. DeLuca, S. Termini. A definition of nonprobabilistic entropy in the setting of fuzzy sets theory. Information and Control 20, 301-312, 1972.

[3] D. Dubois. On degrees of truth, partial ignorance and contradiction. Proceedings IPMU'08, 31-38, 2008.

[4] P. Fishburn. The axioms and algebra of ambiguity. Theory and Decision 34, 119-137, 1993.

[5] C. Fox, A. Tversky. Ambiguity aversion and comparative ignorance. The Quarterly Journal of Economics 110, 585-603, 1995.

[6] J. Goguen. The logic of inexact concepts. Synthese 19, 325-373, 1969.

[7] J. Keynes. A Treatise on Probability. MacMillan, London, 1963. 
[8] F. Knight. Risk, Uncertainty and Profit. University of Chicago Press, Chicago, 1971.

[9] J. Montero, D. Gómez, H. Bustince. On the relevance of some families of fuzzy sets. Fuzzy Sets and Systems 158, 2429, 2442, 2007.

[10] Ch. Osgood, G. Suci, P. Tannenbaum. The Measurement of Meaning. University of Illinois Press, Urbana, 1958.

[11]B. Schweizer, A. Sklar. Probabilistic Metric Spaces. North-Holland, Amsterdam, 1983.

[12] G. Shafer. A Mathematical Theory of Evidence. Princeton University Press, Princeton, 1976.

[13] M. Shubik. Risk, ignorance and indeterminacy. The Quarterly Journal of Economics 68, 629-640, 1954.
[14]E. Trillas. On a model for the meaning of predicates (A naïve approach to the genesis of fuzzy sets). In: Views of Fuzzy Sets and Systems from Different Perspectives (ed.: Rudolf Seising), Springer, 175-205, 2009.

[15] S. Wong, Z. Wang, P. Bollmann-Sdorra. On qualitative measures of ignorance. International Journal of Intelligent Systems 11, 27-47, 1998.

[16] R. Yager. On a measure of ambiguity. International Journal of Intelligent Systems 10, 1001-1019, 1995.

[17]L. Zadeh. Fuzzy sets. Information and Control 8, 338-353, 1965. 